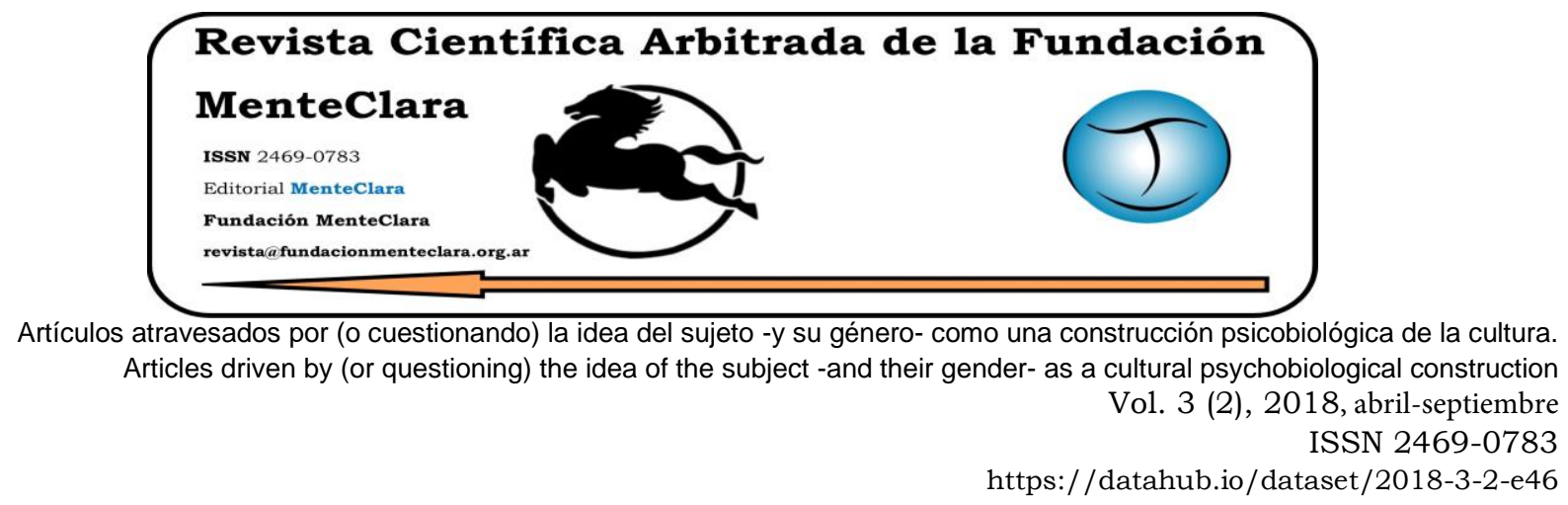

\title{
UN LINGÜISTA EN LA SELVA AMAZÓNICA PERDIÓ SU FE EN DIOS*
}

\section{A LINGUIST IN THE AMAZON JUNGLE LOST HIS FAITH IN GOD}

Anamaria Ashwell aashwell@gmail.com

Benemérita Universidad Autónoma de Puebla. Instituto de Ciencias

Sociales y Humanidades, México.

Cómo citar este artículo / Citation: Ashwell, A. M. (2018). " Un lingüista en la selva amazónica perdió su fe en Dios". Revista Científica Arbitrada de la Fundación MenteClara, 3(2) abril-septiembre 2018, 43-52.

DOI: https://doi.org/10.32351/rca.v3.2.46

Copyright: () 2018 RCAFMC. Este artículo de acceso abierto es distribuido bajo los términos de la licencia Creative Commons Attribution-Non Commercial (by-cn)

Spain 3.0. Recibido: 12/07/2018. Aceptado: 31/07/2018 Publicación online: $19 / 10 / 2018$

Conflicto de intereses: Ninguno que declarar.

* Originalmente publicado en suplemento cultural Saberes y Ciencia de La Jornada de Oriente en Puebla México, enero 2018

\section{Resumen}

Este artículo muestra las dificultades que encontró un evangelizador, convertido posteriormente en antropólogo, para realizar un estudio académico de la etnia Pirahá que habitan la selva amazónica. Después de veinte años de cohabitación con los Pirahá, este evangelizador/antropólogo pudo comprobar lo que los misioneros anteriores reportaron: a los Pirahá les entretenía y hasta interesaba el relato bíblico pero como una historia más. La autora relata las dificultades linguisticas que impide a estos sujetos, sujetados a un lenguaje casi único que contrasta la gramática universal de Chomsky, atribuírle alguna categoría espiritual o salvifica a la palabra de Dios. La autora también describe el regreso del evangelista / antropólogo, unos años después, a la jungla y la forma en que se reencuentra con los Pirahá, pero esta vez sin su fe ni su dios. 


\begin{abstract}
This article presents the difficulties encountered by an evangelist, later converted into an anthropologist, when conducting an academic study of the Pirahá ethnic group living in the Amazon rainforest. Following twenty years of cohabitation with the Pirahá, this evangelist/anthropologist was able to confirm what previous missionaries had reported: the Pirahá were entertained by the biblical account and they were even interested in it but only as a mere story. The author examines the linguistic difficulties that prevent the Pirahá, subjected to an almost unique language contrasting Chomsky's universal grammar, from assigning any spiritual or salvific category to the Word of God. The author also describes the evangelist/anthropologist's return, a few years later, to the jungle and the way he is reunited with the Pirahá but this time without his faith and god.
\end{abstract}

\title{
Palabras Claves/ Keywords
}

Lengua; linguista; idioma primitivo; gramatica; universal; Pirahá; Everett; fe; dios; ateo; Chomsky; Language; linguist; primitive language; grammar; faith; God; atheist 


\section{Introducción}

En la rivera del río Maici, inmersos en la tupida selva brasileña, familias de entre tres y cuatro miembros habitan siete precarias chozas con techos de frondas de palma sostenidos por cuatro postes. A lo largo de las riberas del río Maici y Marmelos, tributarios del Amazonas, entre 300 a 400 otras personas comparten con ellos la lengua y la cultura Pirahá.

Los Pirahá son recolectores, cazadores y ocasionales agricultores de descuidados huertos de mandioca. Pescados y caza constituyen su dieta principal y son también la actividad diaria debido a que los Pirahá no salan ni ahúman la carne, no almacenan alimentos como tampoco la harina de la mandioca salvo para unos pocos dias. Con el tiempo han adoptado la ropa occidental: por más de dos siglos han tenido contacto (aunque desde 1700 los brasileños reportan su presencia) con misioneros, foráneos y no solo brasileños pero sobre todo de manera periódica con comerciantes que navegando el río entablaron con ellos un peculiar, por no decir asombroso, intercambio "comercial". Cuando los Pirahá oyen que se aproximan sus embarcaciones se acomodan en la ribera hasta que los divisan en el río. Y se inicia un intercambio en cantidades arbitrarias, determinadas individualmente, de nueces, maderas, la fruta comestible del couma (o sorva) y goma o caucho crudo que ellos entregan preferentemente a cambio de whiskey y tabaco.

Su sistema de parentesco es de los más simples conocidos entre las culturas del mundo. No hay distinciones de género, se distinguen descendientes por generación y también consanguinidad. Los términos de clasificación solo refieren a parientes conocidos, nunca parentela que murió antes del nacimiento de uno (ego). Son además endogámicos con particularidades: el matrimonio esta permitido entre consanguíneos pero también están permitidas las relaciones sexuales de mujeres con 
foráneos para fines de procrear sin implicar cohabitación. Los hijos son criados en la comunidad Pirahá pero los padres foráneos no adquieren ninguna distinción, privilegio, reconocimiento ni clasificación de parentesco.

A pesar del prolongado contacto con foráneos, sin embargo, los Pirahá se mantienen monolingües y con una lengua que es la única que perduró de la familia lingüística Muran de la Amazonía. Los Pirahá no muestran interés, además, ni indagan sobre los idiomas y las culturas de los foráneos que entran en contacto con ellos y a todas las otras lenguas le nombran "Cabeza chueca" porque "Cabeza enderezada" es el Pirahá que ellos hablan.

En el año 2005 un lingüista, Daniel L. Everett, publicó en Current Anthropology un ensayo titulado: "Condicionantes culturales de la gramática de los Pirahá: otra mirada al diseño del lenguaje humano" ${ }^{1}$ que habria de provocar un debate intenso y prolongado (que cayó más bien como "una bomba" en palabras de Steven Pinker) entre investigadores e investigaciones de antropología lingüística. Brent Berlin, por ejemplo, de los laboratorios de Etnobiologia y Antropologia de la Universidad de Georgia no dudó que "la propuesta de Everett en este ensayo es la más controversial que se ha publicado en la antropología lingüistica en muchos años, quizás desde la aparición del Origen y la Diversificación del Lenguaje de Swadesh en 1971". Investigadores, con grabadoras, protocolos para experimentos, con equipo de sonido, micrófonos y cámaras,

\footnotetext{
${ }^{1}$ Ver Everett, Daniel L. "Cultural Constraint on Grammar and Cognition in Pirahá: Another look at the design features of Human Language". Current Anthropology. Volume 4. No.4 August-October 2005 y la bibliografia sobre la lengua pirahá para una profundización sobre los conceptos linguísticos que en este ensayo solo se introducen. Esa bibliografia, en algunos casos en co autoría con su entonces mujer Keren Everett, profundiza sobre la gramática y la lengua de los Pirahá desde 1983. Sin embargo, fue un artículo anterior, de Peter Gordon de Columbia University, "Numerical Cognition without words: Evidence from Amazonia." (Cognición numérica sin el recurso de palabras: evidencia en la Amazonía), en la revista Science el que realmente alertó a los académicos de la importancia disruptiva que la tésis de Everett introducía en las teorías lingüistas vigentes. (Las traducciones del inglés al español son mías). Ver también, Daniel L. Everett, Don`t Sleep there are Snakes, Vintage, Random House New York. 2008 (existe traducción al español).
} 
principalmente desde Australia, Alemania, Inglaterra y EEUU, descendieron desde entonces sobre los Pirahá en las riveras del río Maici.

\section{Desarrollo}

No es tema específico de este ensayo pero resumiré algunas características de la lengua y la cultura de los Pirahá que provocaron y provocan, este desmedido e intrusivo interés de académicos.

El Pirahá, explicó Everett, es una lengua que carece de numerales, números y términos de contabilidad. Si bien se conocen otras lenguas que contabilizan solo uno-dos-más, en el Pirahá no solo no existe una categoria gramatical para números sino también para formas de dar cuenta de una cuantificación. No tiene términos para "todo", "cada uno", "todos", "mayoría" y "algunos". Ni siquiera nombre para los dedos individuales de la mano aunque ocasionalmente (y Everett explica que solo a partir de insistencias de lingüistas e investigadores) los Pirahá refieren a todos los dedos colectivamente como "palillos de la mano". Y en consecuencia tampoco tiene término para "ultimo". Es una lengua que se compone del más simple inventario de pronombres que se conoce y según Everett, ellos no solo evitan recurrir a éstos sino que él estima que todos los pronombres en Pirahá han sido integrados recientemente desde lenguas Tupi- Guaraní. El inventario fonético del Pirahá esta entre los más reducidos: las mujeres recurren a solo 7 consonantes y 3 vocales y los hombres a 8 consonantes y 3 vocales (equiparable al Rotokas y Hawaiano).

Más significativo aún para la teoría de una gramática universal de Chomsky es que el Pirahá no recurre a incrustaciones (embedding), no incrusta una frase dentro de otra. No tiene tiempo perfecto gramatical y pocos términos para el tiempo (Everett enlista doce) que refieren más bien 
a "otro día", día o noche, luna llena, temporal de agua delgada, temporal de agua gruesa, etc etc. La lengua prioriza más bien términos que expresan experiencias liminales, "situaciones en la que algo entra o sale del horizonte de la experiencia”.

No tienen términos específicos para colores sino solo frases descriptivas cambiantes y no producen arte ni artesanias, no inventan ficciones y no tienen mitos de creación. Tampoco tienen referencias a personajes míticos aunque sí dibujan figuras (Everett las describe como de "palitos") que los Pirahá sostienen son una suerte de espíritus que ellos experimentan directamente. Cuando lingüistas y antropólogos insisten sobre sus origenes ellos solo responden "siempre ha sido así".

Los Everett, Keren y Daniel, después de más treinta años compartiendo la vida con ellos, son los únicos foráneos, los únicos lingüistas, Pirahá parlantes $^{2}$. Es una lengua gutural y prosódica que posee una variedad de sonidos (algunos nasales, otros por aspiraciones o por movimientos de labios), tonos y ritmos que exige también distintos énfasis para que una sola palabra refiera a distintas cosas; y también posee extensiones silábicas que parecen interminables. Una lengua que un foráneo, un lingüista o antropólogo, necesitaría toda una vida entre ellos para aprender. Porque es una lengua que permite obviar vocales y consonantes, intercala sonidos y más bien se canta, tararea y/o silba. Keren Everett sostiene que para aprender el Pirahá hay que aprenderlo

\footnotetext{
${ }^{2}$ Las investigaciones y publicaciones de Marco Antonio T. Gonzalves pueden servir como material suplementario sobre la lingüística y la cultura de los Pirahá a los materiales publicados por Daniel L. Everett. Con la salvedad que Gonzalvez no conoce sino elementos muy rudimentarios de la lengua y la mayor parte de su material etnográfico provino de un informante alejado de su comunidad y fue registrado en portugués.

Ver, Gonzalvez, Marco Antonio Teixeira, O Significado Nome. Cosmología e Nominacao os Pirahá. Sette Letras. Brasil. 1993 y -O Mundo Inacabado. Etnografia Pirahá. Editora UFRJ. Brasil 2001
}

- Nomes e Cosmos: Onosmatica entre os Mura-Pirahá Comunicacao No. 20 Programa de PoGraduacao em Antropología Social. Rio de Janeiro. Brasil 1990. 
cantando. Imitando la manera como los Pirahá abandonan consonantes y vocales y se comunican enteramente por variaciones de tonos y ritmos. Sin sílabas. Daniel Everett le concede la importancia prosódica del lenguaje pero sostiene que hay mucho más que aprender de y en su estructura silábica.

Los Pirahá suenan, dijeron algunos, como pájaros exóticos comunicándose con cuchicheos sonoros y melódicos. Hablan -y cabe decirlo así- una lengua que no parece de humanos.

El Pirahá además no distingue ni valida que exista una "manera correcta" de proceder, no tiene verbos para definir intenciones; y destaca solo la inmediacía de la experiencia. Incluso cuando refieren temores a espíritus malignos que los rondan éstos son espíritus "con sangre", que experimentan y a los cuales "conocen". Everett no reporta ningún término Pirahá que pudiera referir a algo fuera de la inmediata experiencia del que habla; y argumenta convincentemente, con palabras como xibipio, que ellos no piensan ni hablan en abstracciones. Esta palabra se traduce más o menos como "fuera de experiencia", es decir todo lo que ellos no pueden oír ni ver o que alguien vivo no oye o ve. "Cuando alguien camina hacia la doblez del río y lo pierden de vista, los Pirahá no dicen que esa persona simplemente se ha ido sino xibipio- "fuera de experiencia"3. Everett llama a esta atadura lingüística y cultural con la realidad empírica y presente "principio de experiencia-inmediata" (immediacy-of experience principle”) en su disertación sobre la lengua Pirahá. Para los Pirahá no hay un nombrar que venga de "afuera", ni desde un ámbito sagrado, ni desde una residencia divinizada, ni desde un legado originario porque para ellos las cosas aparecen y cuando las perciben, solo en ese momento, las nombran. En realidad los Pirahá, según se desprende del

\footnotetext{
3 Tomado de John Colapinto, “The Interpreter". New Yorker. Abril 2007. Colapinto entrevistó a Everett, durante cinco dias en la comunidad del río Maici.
} 
ensayo de Everett, fuera del tiempo lineal de la metafisica occidental, abiertos a la manifestación de lo que aparece y de lo que no aparece, pareciera que hablan, o más bien cantan, en realidad solo desde lo que les sucede y nada distinto a eso. ${ }^{4} \mathrm{El}$ del Pirahá es un mundo linguístico y cultural que se vive solo en el momento; en el cual nada cambia y nada fue creado, siempre ha sido. Un ordenamiento lógico que no previene ni requiere planeación ni tiene término linguístico de un tiempo futuro y en el que la lengua se expresa únicamente en afirmaciones directas. Solo es real, en otras palabras, lo que observan y oyen.

Los Pirahá, se habría de resignar Everett después de años entre ellos y una vez que accedió a hablar la lengua, son un pueblo, por eso mismo, impermeable, o más bien totalmente insensible, a la palabra del Dios cristiano. Lo comprobaría Daniel Everett porque ni él ni Keren llegaron con los Pirahá a habitar en las riberas del río Maici en la Amazonía brasileña como lingüistas (los estudios universitarios y los trabajos como investigadores de universidades en Inglaterra y EEUU serian posteriores); sino como misioneros cristianos del Instituto Linguístico de Verano (ILV). Una organización evangélica, que se propone diseminar la palabra de Dios traduciendo el Nuevo Testamento a las lenguas de sociedades sin escritura. Y que retoma del cristianismo antiguo no solo lo que algunos describieron como "su avidez por lo inconmensurable", sino que amalgama a "pobres" y "otros" en "hermanos" para extenderles la palabra salvífica desafiando todos los obstáculos. Para el tema de este ensayo basta referir que el ILV exige un entrenamiento realmente extremo y un radical sacrificio personal a sus misioneros, incluso más allá de lo

\footnotetext{
${ }^{4}$ Hay ciertas concepciones y practicas entre los Pirahá, me hizo notar Oscar del Barco, que entrecruzan filosofia y etnología. Este es un ensayo, desde la antropología, que continúa esa conversación. Ver, Oscar del Barco, Exceso y Donación: la búsqueda del Dios sin Dios.

Argentina.2003.
} 
razonable 5 . Daniel Everett, en la selva de los Pirahá, en un contexto cultural monolingüe, con 24 años de edad, casado y con tres hijos, llevaba la encomienda de remontar el fracaso de más de cincuenta años de labor de misioneros que no pudieron trasmitir a los Pirahá el valor espiritual de las historias bíblicas.

\section{Conclusión}

Después de veinte años de cohabitación con ellos, tradujo finalmente el Testamento de Lucas al Pirahá y se dedicó a leerlo entre ellos. El ILV no entrenó a Everett para hacer proselitismo como tal porque consideran suficiente que el "otro" acceda en su lengua a la palabra de Dios. Sin embargo, Everett pronto habria de comprobar lo que los misioneros anteriores reportaron: a los Pirahá les entretenía y hasta interesaba el relato bíblico pero como una historia más. No le atribuyeron ninguna categoria espiritual ni salvífica a la palabra de Dios. Cuando Everett les explicó que era la palabra de Cristo, ellos le preguntaron “¿Conociste a este hombre? "Cuando les decía que vivió hace más de dos mil años los Pirahá reaccionaron de la misma manera cuando notaron que yo utilizaba repelente para insectos"6. En el año 2002, Everett, después de adentrarse "en lecturas filosóficas", atormentado, incapaz de hablar el Pirahá que pudiera trasmitirles la palabra sagrada Dios, sintiendo que "algo estaba mal" y que debía ver todo "desde una perspectiva distinta", abandonó la selva. Cuando regresó unos años después, llegó con los Pirahá sin su Dios. Se había aceptado como ateo ${ }^{7}$.

\footnotetext{
${ }^{5}$ Es útil para entender el cristianismo primitivo que insufla la labor del ILV la obra de Peter Brown, Through the Eye of a Needle: Wealth and the Fall of Rome in the making of Christianity in the West, 350-550 d.C. Princeton U. Press.2012.

${ }^{6} \mathrm{~A}$ los Pirahá los insectos no les molestan, son lo que sucede viviendo en la selva. ¿Por qué ahuyentarlos? ¿A dónde? Un absurdo sin consecuencias.

${ }^{7}$ En el año 2002 cuando Daniel Everett abandonó la selva con su esposa y retornó al trabajo académico en la U. de Manchester. En 2005 se divorciaron. Ella retornó a Porto Velho y a la selva Pirahá para retomar su labor misionera.
} 


\section{Bibliografia}

Brown, Peter "Through the Eye of a Needle: Wealth and the Fall of Rome in the making of Christianity in the West, 350-550 d.C." Princeton University Press 2012

Colapinto, John "The Interpreter". New Yorker. Abril 2007

del Barco, Oscar "Exceso y Donación: la búsqueda del Dios sin Dios" Argentina, 2003

Everett, Daniel L. "Cultural Constraint on Grammar and Cognition in Pirahá: Another look at the design features of Human Language". Current Anthropology. Volume 4. No.4 August-October 2005

Everett, Daniel L. "Don't Sleep, There Are Snakes: Life and Language in the Amazonian Jungle (Vintage Departures)" Random House New York. 2008

Gonzalvez, Marco Antonio Teixeira "Nomes e cosmos: onomástica entre os mura-pirahã" Comunicacao No. 20 Programa de Po-Graduacao em Antropología Social. Rio de Janeiro. Brasil 1990.

Gonzalvez, Marco Antonio Teixeira "O Significado Nome. Cosmología e Nominacao os Pirahá" Sette Letras. Brasil. 1993.

Gonzalvez, Marco Antonio Teixeira "O Mundo Inacabado. Etnografia Pirahá" Editora UFRJ. Brasil 2001

Peter Gordon "Numerical Cognition Without Words: Evidence from Amazonia" Science 15 Oct 2004:Vol. 306, Issue 5695, pp. 496-499 DOI:10.1126/science.1094492 